\title{
Brain Tumor Extraction from MRI Images using MATLAB
}

\author{
Rabia Ijaz ${ }^{1}$, Mohsin Jamil ${ }^{2,3}$ and Syed Omer Gilani ${ }^{1}$ \\ ${ }^{1}$ Department of Biomedical Engineering and Sciences \\ ${ }^{2}$ Department of Robotics and Artificial Intelligence \\ School of Mechanical and Manufacture Engineering (SMME), \\ National University of Sciences and Technology (NUST), Islamabad, Pakistan \\ ${ }^{3}$ Department of Electrical Engineering, Faculty of Engineering, Islamic University \\ Madinah, Saudia Arabia \\ rabiaejaz28@hotmail.com,mohsin@smme.nust.edu.pk_and \\ omer@smme.nust.edu.pk
}

\begin{abstract}
Image segmentation is one of the most challenging techniques in the field of medical image processing. Brain tumor segmentation is emerging technique in this field. This paper refers to the brain tumor segmentation from MR images of patients taken from 'Brain web'. Hybrid methodology is used to extract and detect tumor from MR images using basic concepts of digital image processing. MATLAB is used for this purpose in this paper for applying the proposed algorithm.
\end{abstract}

\section{Introduction}

Brain tumor is abnormal growth of tissues which grow uncontrollably and unchecked by the check points which control the growth of cells normally. Brain tumor can be either primary or metastatic. Tumor that spread from other parts of body to brain is said to be metastatic. Brain tumor is one of the likely causes of mortality among children and adult. In children brain tumors are the cause of one quarter of all cancer deaths. There are about 200 different types of tumors diagnosed in UK each year. Medical resonance imaging is an advance imaging technique which is used to obtain high quality images of different parts of body. MRI is used for anatomical analysis of brain development and brain abnormalities (Logeswari and Karnan 2009). These MRI images are pre-processed so that further morphological operations can be performed on these images for the detection of size, shape and location of tumor. Pre-processing of MRI images is done for noise removal and image enhancement while morphological operations are performed using MATLA B algorithms to separate and detect tumor in brain(Murugavalli and Rajamani 2007). The ultimate goal of segmentation is to extract important features from the image data. The segmentation of tumor from MRI images of brain is time consuming process(Toure, Beiji et al. 2010). MATLAB is fast algorithm used for detection of tumor from MRI images in a very short time. At the end tumor is mapped onto original greyscale image with 255 intensity to make tumor visible in the image.

Received (August 10, 2017), Review Result (November 5, 2017), Accepted (November 20, 2017) 


\section{Methodology}

These are the steps performed on MRI image of brain tumor using MATLAB algorithms.

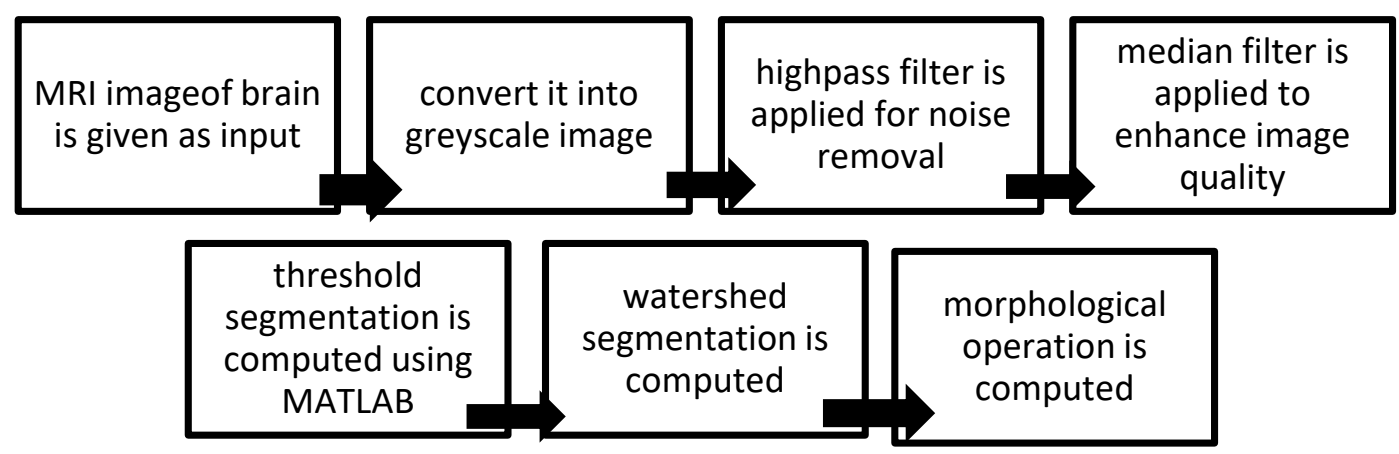

Figure 1. System Level Block Diagram

\section{Grayscale image}

Grayscale image is preferred format for image processing because gray scale images are much less complex and one can talk in detail about contrast, brightness, and edges without considering colors(Roy and Bandyopadhyay 2012). Colors are much more complex since they are composed of three channels that are why image is converted into grayscale before further processing in image segmentation.

\section{Morphological operations}

Top hat filter is used to highlight the sharp peaks and gradients in image. Apply morphological operations in which 'strel' i.e. structuring element of disk shaped is taken and applied on the grayscale image for extracting the tumor from MR image of patients(Deng, Xiao et al. 2009). The tumor obtained in the above step has no clear edges or boundaries but it has somehow sharpened the tumor in the image.

\section{Watershed segmentation}

Watershed image is superimposed over the image after high pass filter has been applied. Watershed transformation is done to segments the cells in an image. It is applied on gray scale image using MATLAB watershed algorithms.

For this work, real world patient data is taken for analysis from 'brain web'. As the tumor in MR images has more intensity than the background that's why it becomes easy to locate and extract the tumor from MR image. 


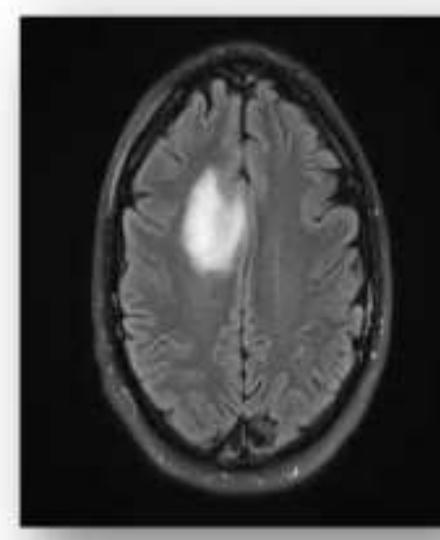

Patil and Bhalchandra (2012)
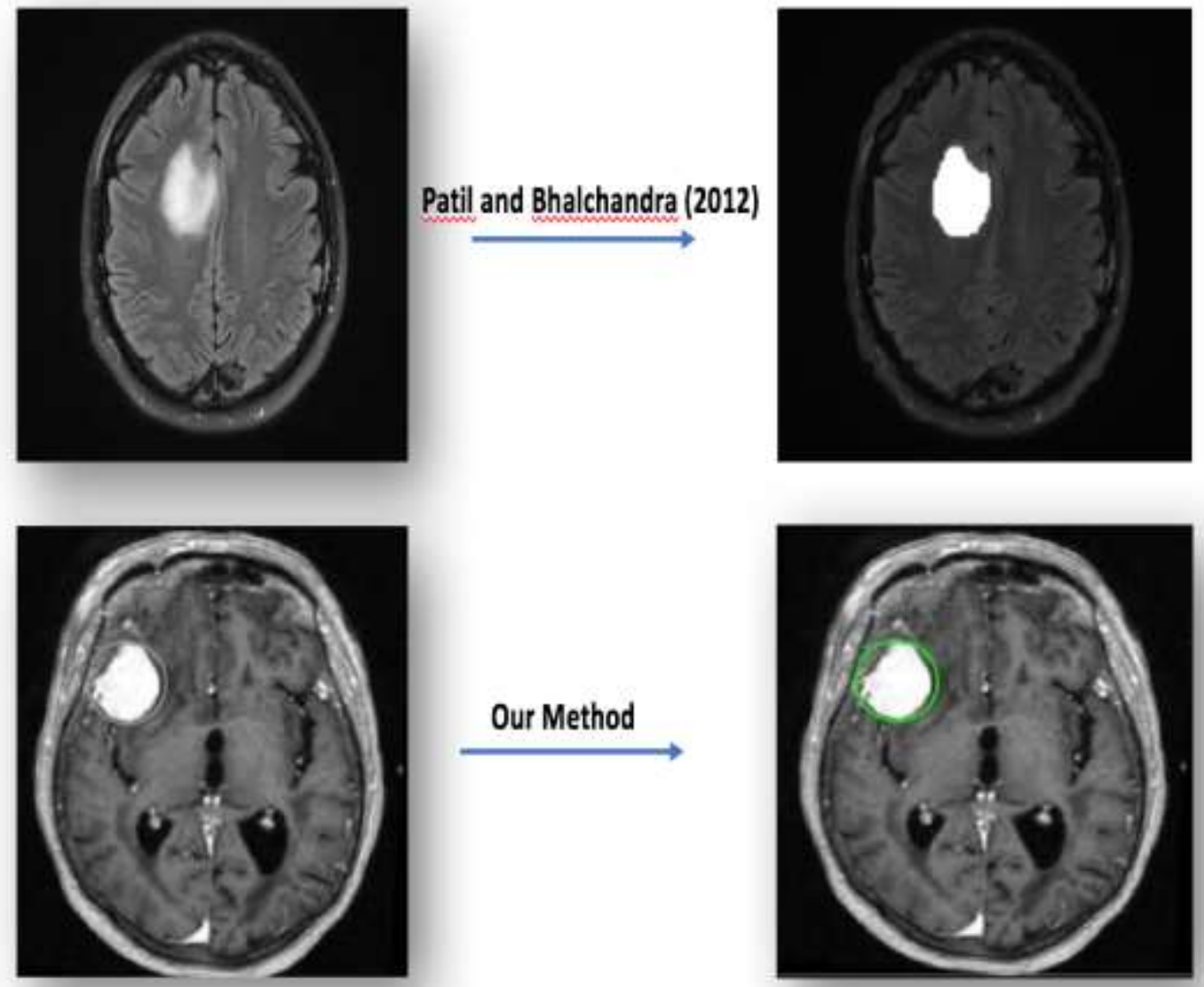

Figure 2. Results of Proposed (Our) Method In Comparison to Existing Method

Previously segmentation was done manually but that process is time consuming and demand intensive labor(Bezdek, Hall et al., 1993). That's why process was automated but still results were not satisfactory.

Some of the brain tumor MR images taken for testing the proposed methodology using the hybrid approach for image segmentation that involves the combination of top hat filter and watershed algorithm(Patil and Bhalchandra 2012). However, their example images are simple case of MRI scans. However, on more complicated images their proposed algorithm failed to generate good results. In contrast, our proposed method shows promising results on more complicated cases. Figure 2 shows the results of both methods

\section{Results}

\section{Pre-processing of MR images}

This is gray scale image of Figure 2. which is converted into gray using MATLAB algorithms.(Kekre, Sarode et al., 2010) this is done once image is resized so that morphological operations can be performed later. 


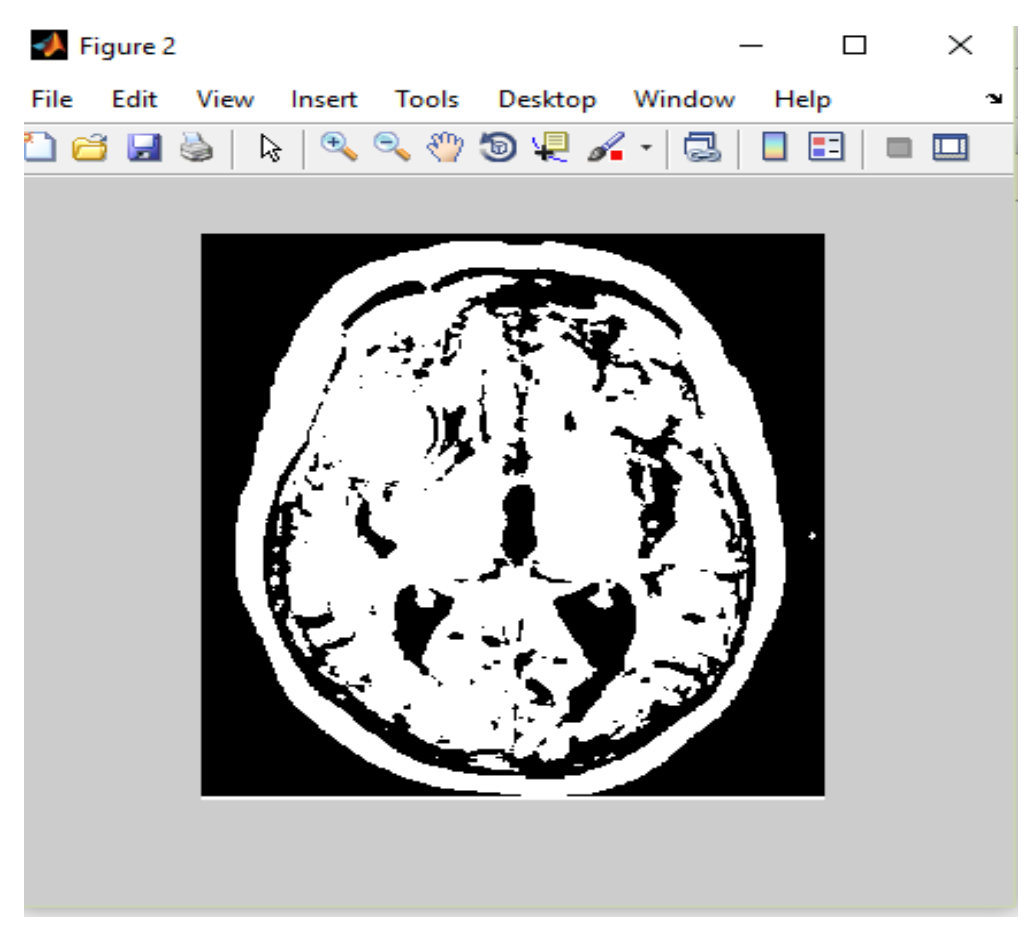

Figure 3. Binarization of an Example Image

\section{Morphological operation}

Image acquired after applying top hat filter kernel transformation which is used to highlight the sharp gradients at peaks.
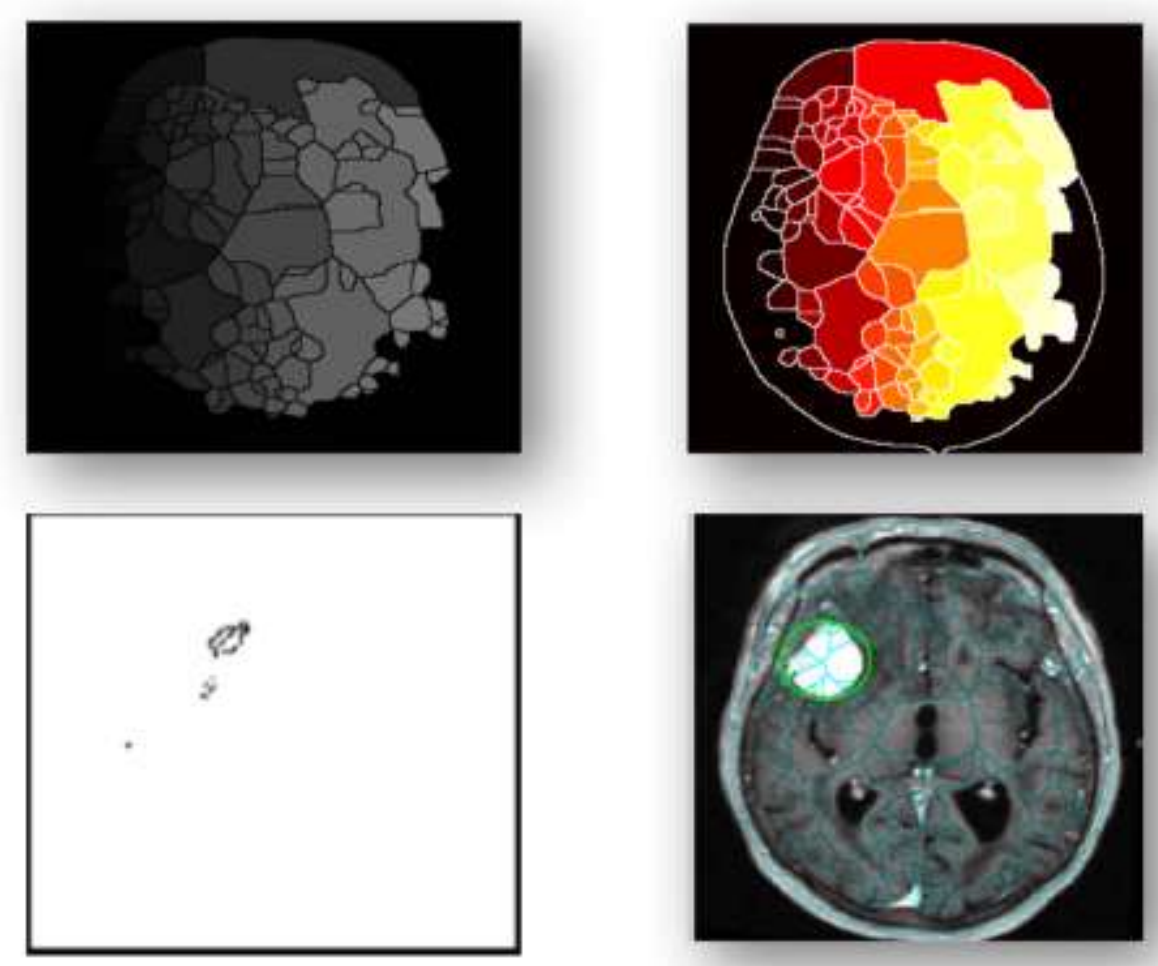

Figure 4. Result of Applying Morphological followed by Watershed 
This is what ideal watershed image for this MR image since we used different algorithm that's why we didn't get the ideal result. Watershed should show cells in the tumor areas of MR but in our case it has shown cells of the whole brain image(Mustaqeem, Javed et al., 2012).

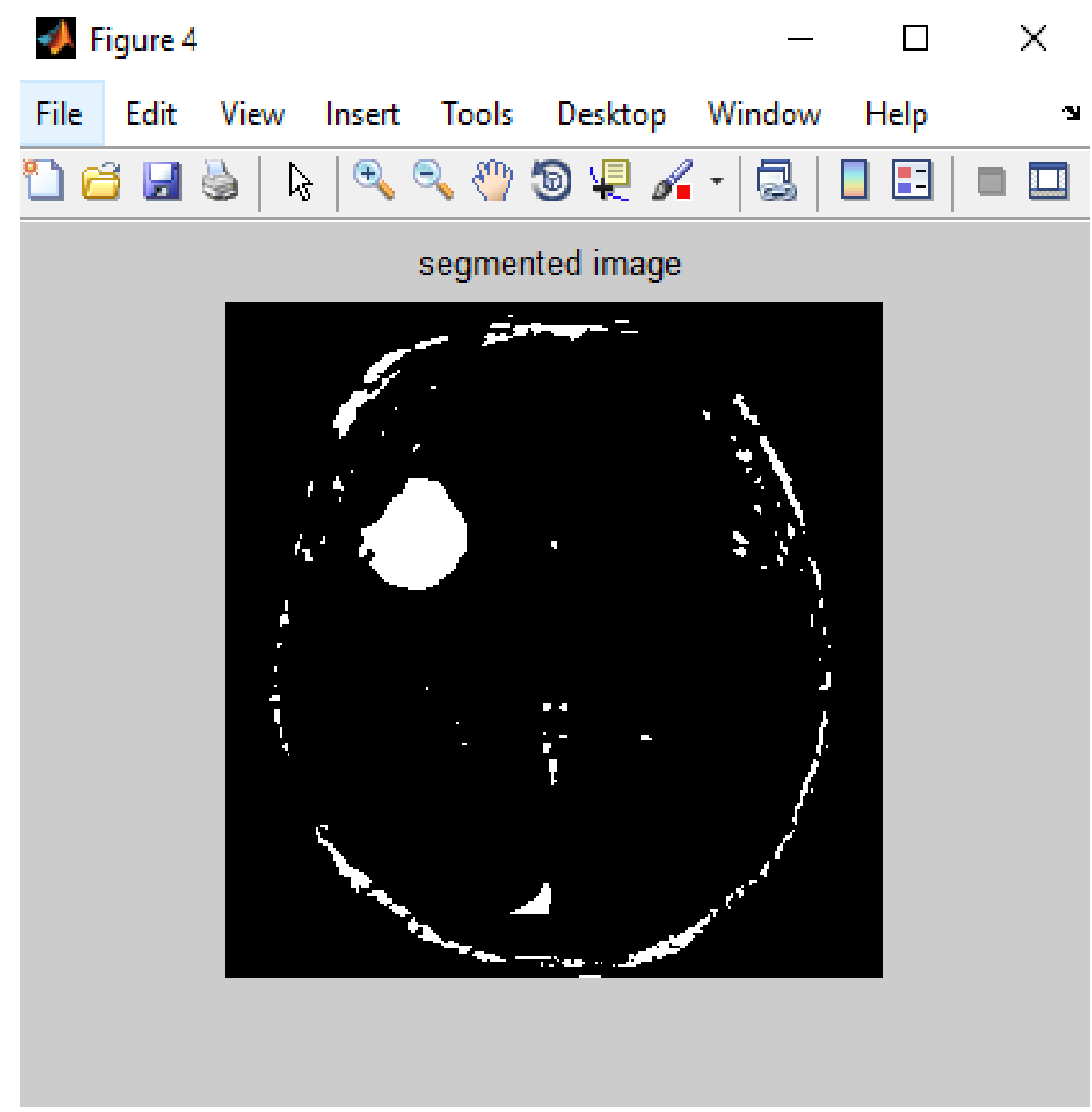

Figure 5. Final Segmented Image

Tumor can be detected in the image. By applying further MATLAB algorithms we can detect the size of this tumor. Since the intensity of tumor is much more than background of image that's the only reason tumor can be detected in MRI(Mancas, Gosselin et al. 2005).For now we can locate the tumor in image precisely.

\section{Future Work}

The above results have been obtained using different algorithms in MATLAB as stated in methodology section. These results have been obtained just to detect the exact location of tumor in the MRI images of real data set of patients. There are number of alternate techniques which can also be used for segmentation. All these techniques use different algorithms in MATLAB for tumor detection(Padmavathi, Muthukumar et al. 2010). There are number of future prospects in this field. This work if elaborated further it can be used to detect tumor size, stage and type using image processing techniques. 


\section{References}

[1] J. C. Bezdek and L. Hall, "Review of MR image segmentation techniques using pattern recognition", Medical physics, vol. 20, no. 4, (1993), pp. 1033-1048.

[2] W. Deng and W. Xiao, "MRI brain tumor segmentation based on improved fuzzy c-means method", Sixth International Symposium on Multispectral Image Processing and Pattern Recognition, International Society for Optics and Photonics, (2009).

[3] H. Kekre and T. Sarode, "Detection of tumor in MRI using vector quantization segmentation", International Journal of Engineering Science and Technology, vol 2, no. 8, (2010), pp. 3753-3757.

[4] T. Logeswari and M. Karnan, "An improved implementation of brain tumor detection using segmentation based on soft computing", Journal of Cancer Research and Experimental Oncology, vol. 2, no. 1, (2009), pp. 006-014.

[5] M. Mancas and B. Gosselin, "Segmentation using a region-growing thresholding", Electronic Imaging 2005, International Society for Optics and Photonics, (2005).

[6] S.Murugavalli and V. Rajamani, "An Improved Implementation of Brain Tumor Detection Using Segmentation Based on Neuro Fuzzy Technique", vol. 1, (2007).

[7] A. Mustaqeem and A. Javed, "An efficient brain tumor detection algorithm using watershed \& thresholding based segmentation", International Journal of Image, Graphics and Signal Processing, vol. 4, no. 10, (2012), p. 34

[8] G. Padmavathi and M. Muthukumar, "Non linear image segmentation using fuzzy c-means clustering method with thresholding for underwater images", IJCSI, vol. 7, no. 3, (2010), p. 35.

[9] R. C.Patil and A. Bhalchandra, "Brain tumour extraction from MRI images using MATLAB", International Journal of Electronics, Communication and Soft Computing Science \& Engineering (IJECSCSE), vol. 2, no. 1, (2012), p. 1.

[10] S. Roy and S. K. Bandyopadhyay, "Detection and Quantification of Brain Tumor from MRI of Brain and it's Symmetric Analysis", International Journal of Information and Communication Technology Research, vol. 2, no. 6, (2012).

[11] M. L.Toure and Z. Beiji, "Advanced algorithm for brain segmentation using fuzzy to localize cancer and epilespy region”, Electronics and Information Engineering (ICEIE), 2010 International Conference On, IEEE, (2010).

\section{Authors}

Rabia Ijaz, she is currently Masters Student at Department of Biomedical Engineering and Sciences within NUST School of Mechanical and Manufacturing Engineering (SMME). Her research interests include biomedical imaging.

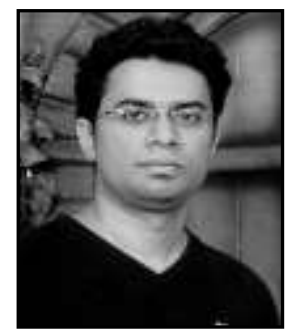

Syed Omer Gilani, he is currently an assistant professor at National University of Sciences and Technology (NUST), Pakistan. He earned his Ph.D. in electrical and computer engineering from National University of Singapore in 2013 and MSc degree in computer engineering from Sweden in 2006. Between 2006 and 2008, he worked at Interactive Multimedia Lab (Singapore). His research interests include human-machine interaction and networking and actively consults for industry on various projects.

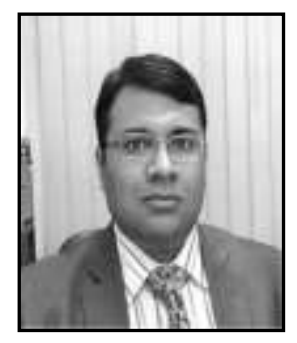

Mohsin Jamil, he is currently assistant professor and Dy Head of Department at National University of Sciences and Technology (NUST), Islamabad, Pakistan. He received his BEng Industrial Electronics degree from NED University, Pakistan, in 2004. He is MSc Electrical Engineering degree holder from Dalarna University Sweden and National University of Singapore in controls and automation. He received $\mathrm{PhD}$ Degree from University of Southampton, UK. His research interests include digital control design and smart grid technologies. He is author of several IEEE publications. 